WIENER SLAVISTISCHES JAHRBUCH, Band 57/2011, 179-192

(C) 2011 by Österreichische Akademie der Wissenschaften, Wien

Tone SmoleJ

\title{
Wien in der slowenischen Literatur
}

Die österreichische Literaturgeschichte hat das Motiv Wiens in der slowenischen Literatur bereits behandelt. Maria Vera Claricini (1996) hat im umfangreichen Sammelband Wien als Magnet? eine Abhandlung über Cankars Wien veröffentlicht. Innovativer ist die Studie von Stefan Simonek (2004), der vor allem die Welt der Wiener Parks bei Cankar analysiert. Der wichtigste slowenische Literaturwissenschaftler, der Cankars Verhältnis zu Wien untersuchte, war jedoch France Bernik (1998). Während sich die Forscher bisher vor allem auf Cankar konzentrierten, will die vorliegende Studie noch andere slowenische Autoren vorstellen, die Wien beschrieben haben, und somit einige Konstanten in der slowenischen Darstellung dieser Stadt präsentieren.

\section{Spätromantiker Und ReAlisten}

Die erste Beschreibung Wiens findet man bereits in der ersten slowenischen Erzählung Sreča v nesreči (Glück im Unglück) von Janez Cigler. Pavle Svetin begibt sich nach Wien und sieht schon von weitem den hohen Turm des Stephansdoms, „der zwischen den hohen Gebäuden hervorragt und von dessen Höhe er schon so viel gehört hat" (Cigler 1936: 69). In der berühmten Kathedrale fasst der Held den Beschluss, Priester zu werden.

Ähnlich wie Ciglers Svetin wurden der Turm und der Stephansdom - ,ein herrliches Andenken der gotischen Baukunst" (Trdina 1948: 64) - auch von Janez Trdina bewundert, der im Herbst 1850 nach Wien kam, um Geschichte und Geographie zu studieren. Aus seinen späteren Memoiren (Spomini) weiß man, dass er sich die Hauptstadt vor seiner Ankunft anders vorgestellt hatte und dass er von den städtischen und vorstädtischen Palästen Wiens enttäuscht war, da sie ,aus unterschiedlichsten Stilen und Unstilen“ zusammengewürfelt seien (ibid.: 64). Den berühmtesten Palast beschrieb er folgendermaßen: „Schönbrunn ist für Kinder und Weiber, die sich gerne Goldfische und Affen anschauen“ (ibid.: 70). Er kritisierte auch die Lage der Kaserne Arsenal, von der aus man die widerspenstige Stadt beschießen und sogar zerstören könnte. Er war viel mehr von den städtischen Parks angetan, vor allem 
vom Belvedere und von Schwarzenbergs Garten, wo er zu studieren pflegte. In dieser Zeit war dieser einst sehr wichtige Park bereits vollkommen verlassen und ungepflegt. Vielleicht leistete ihm dort manchmal Matija Valjavec Gesellschaft, der ein Jahr nach Trdina nach Wien kam, um klassische Philologie und Slawistik zu studieren. In seinen „Wiener Terzinen“ (Dunajske tercine) besingt er auch den Garten Schwarzenbergs:

Na vrtu kneza Švarcenberga v hladu Sprehajajo se dunajčanske rože, Zadoščevaje srčne želje gladu.

Jaz tudi vrinem se med ljudstva množe,

Ker polni cvet $\mathrm{z}$ veseljem ogledujem,

In kjer jih več je, lepših vidiš lože

In veš, lepote rad ne prezirújem. (Valjavec 1900: 16) ${ }^{1}$

Im Jahr 1855 lebte Fran Levstik nach dem Hinauswurf aus dem Priesterseminar in Olmütz einige Zeit in Wien. Drei Jahre später situierte er die Handlung seiner berühmten Erzählung Martin Krpan gerade in der Metropole der Monarchie. Für uns ist jedoch sein (wahrscheinlich autobiographisches) Gedicht von Interesse, das den Titel Meniška cerkev in dunajski vrt (Die Mönchskirche und der Wiener Garten) trägt, in welchem man auch folgende Strophen findet:

Po Dunaju vrtov je lepih dovolj, po vrtih so drevja hladne vrsté; med drevjem podobe kamnite stojé, po ribnikih plavajo ribice zgolj.

Po dunajskih vrtih ljudi je dovolj, bogatih, zalih, gosposkih ljudi; veselje iz lepih jim sije oči, tam iščejo kratkega časa najbolj.

Tam polno deklet je vsaki dan, ki z ljubimi sem ter tje hodijo, prijazno pod pazho se vodijo; pred soncem so veje strop jim hladan. ${ }^{2}$ (Levstik 1952: 65-66)

${ }^{1}$ Prosaübersetzung: Im kühlen Garten des Fürsten Schwarzenberg / gehen Wiener Blumen spazieren / um den Hunger des Herzenswunsches zu stillen. / Auch ich drängle mich zwischen die Menschenmengen, / da ich selig die volle Blüte bewundere, / und wo es mehrere gibt, erblickt man leichter schönere, / und du weißt ja, dass ich die Schönheit nicht verschmähe.

2 Prosaübersetzung: In Wien gibt es genug schöne Gärten, / in den Gärten kühle Baumreihen; / zwischen den Bäumen stehen steinerne Statuen, / nur die Fischlein schwimmen in den Teichen. // In den Wiener Gärten gibt es genug Leute, / reiche, schöne, stattliche Leute; / Freude leuchtet aus ihren schönen Augen, / dort suchen sie meist ihren Zeitvertreib. // Jeden Tag gibt es dort viele Mädchen, / die mit ihren Liebsten hin und her spazieren, / freundlich unter dem Arm geführt; / die Äste eine kühle Decke, die sie vor der Sonne schützt. 
Es handelt sich um ein Gedicht von einem ehemaligen Mönch, wie auch Levstik selbst einmal einer war, der im Park eine schöne Wienerin mit zwei Kindern beobachtet und danach traurig nach Hause geht. Elf Jahre später schrieb Levstik sogar ein Gedicht mit dem Titel Dunajčanki (An die Wienerin), in dem er jedoch seiner slowenischen Partnerin Treue verspricht (Levstik 1948). Der Park, in dem das lyrische Subjekt den Wienerinnen nachstellt, bildet demnach in den fünfziger Jahren des neunzehnten Jahrhunderts eine wesentliche Komponente des slowenischen Bildes der Stadt Wien.

Ungewöhnlich wenig oder fast keine Beschreibungen Wiens kann man bei Josip Stritar vorfinden, der den größten Teil seines Lebens in der österreichisch-ungarischen Metropole bzw. in ihrer Umgebung verbrachte. Er kam im Wintersemester 1855/56 nach Wien, um klassische Philologie zu studieren. In einem Brief an seinen Bruder Andrej beschrieb er am 9. 10. 1855 die Stadt folgendermaßen:

Die Stadt Wien ist an und für sich nicht viel größer als Ljubljana und zählt um die 1200 Häuser. Rund um die Stadt gibt es eine Mauer (Bastei), die man besteigen kann; durch diese Mauer führen in die Vororte, die rund um die Stadt liegen (34 an der Zahl), 14 kleinere und größere Tore - am schönsten ist das „Burgthor“. Nach der Bastei kommen aber nicht sofort die Vororte, sondern es erstreckt sich rund herum das sog. „Glacis“, was nichts Anderes ist als ein ungefähr ein achtel Stunde breiter Raum, der von Bäumen umsäumt wird - fast wie die Sternallee von Ljubljana. Bin schon viel in der Stadt herumgekommen. Habe den Turm des Heiligen Stephan gesehen und die Kirche - wirklich großartig - jedoch eigentlich nicht zu groß. (Stritar 1957: 227-228)

In diesem Brief greift Stritar bei den Beschreibungen auf etwas ungewöhnliche Vergleiche mit Ljubljana zurück. Es ist interessant, dass Stritar fünfzehn Jahre später in seinen Popotni spisi / Reisebriefe (Zvon, 1870) den Bau der Gebäude am Ring beschreibt, nachdem die Mauer abgerissen worden ist. Er bedauert, dass die Stadtverwaltung dort keinen Platz errichtete. Er lobt jedoch das Parlament im griechischen Stil, das ,eine architektonische Perle sein wird“ (Stritar 1955: 400), obwohl er es selber auf einer höherliegenden Stelle erbauen würde, damit man es von weitem sehen könnte. Stritar hatte jedoch Bedenken hinsichtlich des ungewöhnlichen gotischen Rathauses, das zu teuer zu werden drohte. Würde man das Geld unter die Bewohner Krains verteilen, würde jede Familie 100 Gulden bekommen. Da er aber kein Kommunist sei, überlasse er das Urteil dem Leser. Das sind eigentlich die einzigen deutlicheren Beschreibungen Wiens in Stritars Werk. Das Adjektiv „Wiener“ in den Titeln Dunajski soneti (Wiener Sonette), Dunajske elegije (Wiener Elegien) und Dunajska pisma (Wiener Briefe) bezeichnet lediglich den Entstehungsort. Obwohl Stritar gerne andere Städte (Paris, Montmorency) beschrieb, erwähnte er die eigene Stadt kaum.

Ein Jahrzehnt nach Stritar studierte auch Josip Jurčič klassische Philologie in Wien. Obwohl seine schriftstellerische Feder die Stadt Wien nie dargestellt hat, hat er als Journalist mindestens zweimal für die Zeitschrift Slovenski narod über die Ereignisse im Parlament berichtet, das damals noch ein Holzgebäude am Schottentor war. Das Geschehen verglich er humorvoll mit der Hölle Daniels (Jurčič 1982). 
Während sich Jurčič, nachdem er das Studium abbrach, als Journalist durchschlagen musste, konnte sein Freund Fran Levec im Jahr 1868 noch einige unbeschwerte Stunden als Student genießen. In der Korrespondenz mit seiner späteren Frau Jerica findet man folgende gekonnte literarische Beschreibung eines Sonntagnachmittages im Mai im Prater:

Wer etwas sehen will, wer den Wiener kennenlernen will, wer wissen will, wie man in Wien lebt - der muss in den Prater! Vom ärmsten Wiener Arbeiter, der barfuß und in zerrissener Kleidung in den Prater latscht, bis hin zum höchsten Aristokraten, der sich mit sechs Pferden dorthin kutschieren lässt - alles eilt in den Prater, alles freut sich sonntags im Prater - der Bettler im Wurstelprater, der Reiche im großen Prater. Der Fremde, der zum ersten Mal in dieses Wiener Paradies eintritt, muss wirklich aufpassen, um dort nicht sein Auge und Herz, oder noch etwas anderes zu lassen! (Levec 1967: 30)

Erst fünfundzwanzig Jahre später besuchte Fran Govekar den Prater, unser erster ausgeprägter „Wiener“ Autor.

\section{Fran GoveKar}

Govekar beschrieb die Feste im Prater in seiner Novelle $O$ te ženske! (Ach, die Weiber!). Drei slowenische Studenten langweilen sich an einem winterlichen Sonntag und überlegen, was sie im Prater machen könnten:

Oh, wäre es jetzt kein Winter, würden wir an so einem freien Tag in den lebhaften, lustigen Prater eilen, wo alles witzelt und lacht, wo man alles ,halb umsonst" sieht und hört. Dort würde man sich vielleicht auf die Rutsche setzen, und für fünf Münzen in drei Minuten, ,über Berg und Tal“ flitzen, - man würde sich vielleicht noch einmal das berühmte „Panoptikum“ anschauen, daß „nur für Erwachsene“ ist, - vielleicht würde man sich schnell auf einer Leiter fotografieren lassen, einer über dem anderen, der dritte sitzend, vielleicht würde man beim „wilden Ritt" auf dem einen oder dem anderen „Ringelspiel“" mitmachen, oder die Kraft seiner Fäuste messen, indem man den Strohneger ohrfeigt [...]. (Govekar 1897: 266)

Govekar studierte zwischen 1892 und 1897 Medizin in Wien. Da er sich in der österreichisch-ungarischen Hauptstadt intensiv für Theater, Literatur und vor allem für die Kaffeehäuser zu interessieren begann, beendete er sein Studium nie. Sonst aber schilderte er sein Leben im Jahr 1896 einem Freund folgendermaßen:

Die letzten zwei Wochen haben wir durch und durch gezecht. Die Zentrale des Zechens und die Endstation war natürlich der „Lustige Affe“ oder „Zu den 3 Knödeln“. [...] In der letzten Woche habe ich an keinem Tag mehr als 3-4 Stunden geschlafen. Ich habe zwei junge Frauen und ein Fräulein herumgeführt. Alle drei bewundern mich! [...] Sie waren überaus lebhaft und gar nicht brav. Auch in das - Hurenhaus musste ich sie führen, da sie unbedingt auch das „Nachtleben“ kennenlernen wollten. Wir waren im „Eldorado“, im Café „Seitz“ und im „Orfeum“. Überall hatten wir grand-Spass. (Govekar 1978: 14-15)

Im selben Jahr machte Govekar mit dem Roman $V$ krvi (Im Blut) auf sich aufmerksam, einem naturalistischen Roman, der in der Zeitschrift Ljubljanski zvon erschien. Der Schlussteil des Romans spielt in Wien, und der Schriftsteller beschreibt die Stimmung um Mitternacht in einem Kaffeehaus in der Alserstraße folgendermaBen: 
Im Saal rauschte es, es raschelte wie in einem Bienenstock vor dem Schwärmen. Alle Tischchen waren okkupiert, jeder Stuhl besetzt, alle Sofas und Armstühle dort an den Wänden waren voll - Frauen und Männer. Und alle waren bestens gelaunt. Lachen, Gelächter, kurzes, anzügliches Singen, Knallen der Champagnerflaschen, Klirren der Gläser ... all das mischte sich mit dem schweren Duft der feinen Zigarren und Zigaretten, mit dem berauschenden Duft des aufdringlichen Patschoulis, Moschus und - poudre de riz. (Govekar 1896a: 716)

Für unser Thema ist jedoch wahrscheinlich die Novelle Vzor (Das Vorbild) am interessantesten, da sie einige detaillierte Wiener Impressionen enthält. Schauen wir uns die Beschreibung des Volksgartens an:

Es war im Mai, wenn Wien so schön ist, so warm und so hell. Im Volksgarten blühten riesige violett-rote Fliedersträucher an den unzähligen weißen Kieswegen und verbreiteten ihren prächtigsten Duft; Rosskastanien rühmten sich mit jungem, weichem, hellgrünem Gefieder; die große Fontäne spritzte ihren silbernen Strahl in unermessliche Höhe und zerfiel fallend in Millionen und Millionen von vereinzelten Perlen. Die Spatzen kreischten, stritten sich, badeten und spielten. Eine leichte Briese rauschte in den Baumwipfeln und im Gebüsch, doch oben am azurblauen Himmel, rein wie eine Jungfrauenseele, lächelte unsere gutmütige Sonne. Auf den Wegen, Kieswegen und kleinen Pfaden wimmelte es von freudigen, schönen, eleganten Menschen. Oh, wie viele undurchdringlich tiefe, schwarze, mit solcher Wärme blickende, geistreiche, witzige, träumerische Augen ... oh, wie viele herrliche Lippen, wie viele klassische Näschen, Kinnchen und Öhrchen ... oh, wie viele beeindruckend wohlgebaute Körper, wie gemacht für die Liebe, sah man dort! - Und überall die Freude - überall das Lachen - das Leben. Dort hinten, in der Nähe der Statue der so feinfühligen Dichterin Sappho, dort in der Stille der Bäume spielte und spielte die Blaskapelle der Armee, die den ganzen Garten mit einem Meer von süß aufregenden, die Seele erhebenden und mit dem Herz spielenden Stimmen erfüllte. Und die Leute spazierten, sangen halb laut nach der Musik, im Takt des lebhaften Walzers, halb gehend, halb tanzend und lachend, mit koketten Schritten und Blicken, froh, glücklich, selig ... Hei, ihr Maitage in Wien, hei, ihr seligen Stunden zwischen dem blühenden, duftenden Flieder und dem fröhlich singenden schönen Wienervolk, es vergisst euch nie, wer euch einmal erblickt und mit solch glücklichem, randvollem Herzen genossen hat, wie mein Freund - der Idealist Janko! (Govekar 1896c: 683)

Neben Blumen und erneut auch Wienerinnen versäumt es Govekar nicht, auch Fernkorns Fontäne und Sappho zu erwähnen, die zwar im Park keine Statue besitzt, doch kann man ihr Bildnis auf der Reliefwand neben Kundmanns Denkmal des Dramatikers Grillparzer finden. Im Volksgarten lernt Janko, ein junger Philosophieabsolvent, eine schöne Anstandsdame kennen - die Tschechin Elza. Um sie zu beeindrucken, ist er bereit, mit ihr sogar in ihrer Muttersprache zu reden, da das Mädchen keine Kontakte zu den tschechischen Mitbürgern in Wien pflegt. An diesem Tag spaziert der überglückliche Janko am Graben entlang:

Der breite Graben wurde vom modernsten, reichsten, schönsten, anzüglichsten, aber auch angeberischsten Publikum bevölkert, das auf dem breiten Gehsteig in zwei Reihen hin und her spazierte. Und diese fröhlichen, ausgelassenen, erhaben ernsten, gigerlmäßig blasierten, in allen Sprachen der Welt plaudernden Menschen schienen Janko - lauter Freunde. An dem Abend liebte er sie alle, er würde am liebsten jeden umarmen und ihm von seinem Glück erzählen. (Govekar 1896c: 690)

Mit der Beschreibung Wiens hängt auch ein Besuch des Volkstheaters zusammen, das Govekar, sein regelmäßiger Besucher, folgendermaßen schildert: 
Es kamen Herren in dunklen Salonröcken, mit Handschuhen, mit Blümchen im linken Knopfloch und mit einem Fernglas über der Schulter. Sie begleiteten Damen in hellblauen Abendroben, tief ausgeschnitten auf der Brust- und Rückenseite, mit Kurzärmeln und Handschuhen, die ihnen über den Unterarm hinaus reichten. Fast jeder hing auf einer zarten Kette ein elegantes Binokel oder Lorgnet um den Hals. Uniformierte Diener verbeugten sich vor ihnen, führten sie, vor ihnen gehend, nahmen ihnen die Eintrittskarten ab und öffneten für sie die Saaltüren. Überall herrschte Rauschen, nervöses Laufen, Fragen, Antworten, Klirren der Gläser, Gleiten, Krach und Lachen. (Govekar 1896c: 690)

An dem Abend wird das Drama Heimat von Hermann Sudermann aufgeführt, das von Govekar als eine furchterregende Darstellung der Hinterlist und Heuchelei beschrieben wird. In diesem Drama sollte die berühmte Schauspielerin Adele Sandrock als Magda auftreten, die Govekar in seinem Werk als ,,unübertreffliche Künstlerin" lobt. Wahrheitsgemäß muss man jedoch hinzufügen, dass das Wiener Publikum die Sandrock damals nicht sehen konnte, da sie diese Rolle als Gast nur im Jahr 1894 im Theater von Bad Ischl interpretierte (Balk 1997: 146). ${ }^{3}$ Sudermanns Drama erzählt die Geschichte der berühmten Sängerin Magda, die wegen einer Liebesaffäre, aus der auch ein Kind hervorging, von ihrem Vater verstoßen wird. Der ehemalige Liebhaber würde jetzt die Sängerin heiraten, aber nur, wenn sie das gemeinsame Kind verschweigen würde, das er jedoch später adoptieren würde. Die Motive des Dramas von Sudermann sind auch mit dem weiteren Schicksal von Govekars Helden verbunden. Die listige Elza überzeugt ihn, ein Waisenkind zu adoptieren, das in Wirklichkeit ihr unehelicher Sohn ist.

Govekar war ein begabter Beobachter, doch hatte er kein Gespür für die inhaltliche Entwicklung der Geschichten, die er oft sogar von Zola übernahm. In der Novelle Socijalist! (Der Sozialist!), die auch unter dem Einfluss von Zolas Novellenzyklus Comment on meurt (Wie man stirbt) entstanden ist, versuchte sich Govekar - bisher eher ein Darsteller der Wiener Parks und Theater - auch in der Schilderung des armen 17. Wiener Bezirks Hernals, einer Gegend, die er gut kannte, da er am Anfang seines Studiums in der Hernalser Hauptstraße wohnte und sich angeblich sogar in der Volksküche zusammen mit Arbeitern und armen Leuten ernährte (Govekar 1896b). Der Ausschnitt aus dem Arbeiterleben Der Sozialist! schildert die tschechische Familie Ružička, die in Hernals wohnt. Vater Jan verliert seine Arbeit als Lokführer, weil er in der Delegation ist, die vom Eigentümer der Fabrik eine Lohnerhöhung fordert. Die Armut verursacht den Tod seines talentierten Sohnes. Wegen der Passage, in der der Vater in einem kleinen Sarg seinen Sohn zum Friedhof von Hernals trägt, haben in Ljubljana angeblich alle Leser geweint, sogar der Herausgeber der Zeitschrift Zvon (Govekar 1978: 17). Am Ende vergiftet sich die Familie mit Kohlenmonoxid.

${ }^{3}$ Sandrock ist als Magda in Wien erst in den Jahren 1898 (Raimundtheater) und 1903 aufgetreten (Deutsches Volkstheater; Balk 1997: 147, 148). Sudermanns Drama wurde jedoch in Ljubljana im Jahr 1896 aufgeführt. 
Govekar, der Schilderer der Armut in Hernals, wird bald von Ivan Cankar, dem Dichter Ottakrings, abgelöst.

\section{IVAN CANKAR}

Vier Jahre nach Govekar und vier Jahrzehnte nach Stritar kam im Jahr 1896 Ivan Cankar nach Wien, der sich zuerst an der Ingenieursschule der K.u.K Technischen Hochschule immatrikulierte. Seinem jüngeren Bruder Karl, einem späteren Priester, beschrieb er die ersten Eindrücke in Wien in einem Brief vom 4. 11. 1896 folgendermaßen:

Ich war vollkommen betäubt! Du kannst dir diese Schönheit gar nicht vorstellen - z. B. am Ring. Ein Palast neben dem anderen. Das Parlament in wunderbarem ionischen Stil, das Rathaus im gotischen, die Oper und das Burgtheater im Stil der Renaissance, das Kunsthistorische und das Naturhistorische Museum, - aber es ist müßig aufzuzählen! Ich hatte mir zu Hause wie ein Kind vorgestellt, wie wunderbar es hier sein muss, - doch wer hätte gedacht, dass es zehnmal schöner ist! In Ljubljana gibt es wirklich ein paar Gebäude, die auf Deutsch als ,hübsch“ bezeichnet werden, hier jedoch imponiert an allem der überwältigende Stil. (Cankar 1970: 18)

Der Brief, in dem Cankar seinen ersten Kontakt mit der Großstadt schildert, hat sicherlich Gemeinsamkeiten mit Stritar. Aus beiden Briefen ist ersichtlich, wie die beiden Autoren sich die Stadt vor der Ankunft vorgestellt haben, in beiden findet man Vergleiche mit Ljubljana. Cankar war vom Ring begeistert, Stritar war jedoch bei seiner Umgestaltung, wie wir schon gesehen haben, eher zurückhaltend oder sogar kritisch. Cankar erwähnt den Ring später poetisch am Anfang seiner Vignetten: „Der Wind wehte Staubwolken den Ring entlang“ (Cankar 1969: 7). Sonst aber ist am Ring sein Zyklus wenig bekannter humorvoller deutscher Gedichte über den Gaskandelaber verortet:

Ein Gaskandelaber steht einsam

vor der Oper am Opernring.

Ihn schläfert; die Tramwayglocken,

Die machen: kling-kling-kling.

Da sagt der Gaskandelaber:

das ist doch wirklich schön,

dass ihr da fortwährend klingelt,

ich möchte schon schlafen gehn! (Cankar 1968a: 139)

Neben dem Ring haben Cankar auch die Wiener Parks beeindruckt, die er ebenfalls in seinem Brief an den Bruder erwähnt: „An vielen Straßen gibt es Alleen und lauter Gärten, in denen es Bänke gibt und Promenaden, und das mitten in der Stadt (so z. B. ,Volksgarten“, ,Stadtpark' usw.)“ (Cankar 1970: 18). Im Volksgarten bewunderte er z. B. die Statue Grillparzers von Carl Kundmann mit Reliefen aus seinem Werk, das - wie gesagt - bereits Govekar inspirierte. In die Wiener Parks ging er offensichtlich auch später gerne, da er einige Jahre danach am 22. 3. 1899 an seinen Bruder schrieb: „In Wien haben wir schon einen prächtigen Frühling; die Sonne 
scheint wie im Mai und im Stadtpark treiben schon die Mandelblüten. Das ist schon irgendwie tröstlich." (Cankar 1970: 50)

Bereits Stefan Simonek (2004: 85) stellt fest, dass im Gegensatz zum Autor seine Figuren keinen Zugang zu den städtischen Parks haben, sondern daraus ausgeschlossen sind. Ein gutes Beispiel dafür ist das Mädchen Anka aus der Erzählung Iz Predmestja (Aus der Vorstadt) aus der Sammlung Knjiga za lahkomiselne ljudi (Ein Buch für Leichtfertige). Das Mädchen wurde in „der Vorstadt geboren, die in den Rauch großer Fabriken und in den Staub der ungereinigten Straßen eingehüllt ist" (Cankar 1968b: 135), deswegen muss es bereits mit vierzehn Jahren in einer Krawattenschneiderei arbeiten. Eines Tages schickt es die Meisterin Geld holen, doch statt sofort zurück zu kehren, geht es in die Stadt und kommt so sogar zum Park: „Da erschien vor ihren Augen etwas Wunderbares: [...] da gab es Bäume, wirklich grüne, prächtige Bäume, so anders als die verstaubten und kranken Kastanien, die in den städtischen Parks schlummerten. Auf Anhieb hörte die Stadt auf, die Straßen gingen in wunderschöne Alleen über und es erschienen kleine niedliche Kieswege" (Cankar 1968b: 141). In der gleichen Sammlung findet man auch die Novelle Pred ciljem (Vor dem Ziel), in welcher die Hauptfigur ebenfalls davon träumt, einmal einen Palast und einen wunderschönen Garten zu besitzen. Die Novelle ist auch deshalb interessant, da sich Karl Jereb aus dem proletarischen Milieu in die Wiener Staatsoper begibt. Zwischen den teuer gekleideten Damen und den kahlköpfigen Kaufmännern fühlt er sich zwar nicht wohl, doch die Musik besänftigt ihn: „Doch war es ihm angenehm, daß man spielte, sprach und sang; sehr ergötzten ihn die Stimmen der Violinen, die manchmal klar und triumphierend aus dem Orchester erklangen. Eine träge, süße Empfindung umfasste ihn ganz; sie legte sich ihm sogar aufs Gesicht, und seine Lippen lächelten. Es war ihm, als würde er baden, als würde er bis zu den Lippen in reinem, lauwarmem Wasser liegen, schläfrig, angenehm faul" (Cankar 1994c: 64-65). Neben dem Stadtpark und dem Volksgarten war also auch die Oper am Ring ein wichtiger Zufluchtsort, ein locus amoenus für Cankars Figuren.

Mit der Wiener Oper hängt auch die Erzählung Mimi zusammen. Ähnlich wie Anka wohnt auch diese Heldin in der schmutzigen Vorstadt: „Über den Dächern rankt Rauch aus Fabriken, und gehst du die Gasse entlang, fällt dir Ruß ins Gesicht.“ (Cankar 1995a: 7). Der Erzähler lädt das Mädchen anlässlich ihres Namenstages in die Oper ein. Sie begeben sich auf den Weg ins Zentrum, wo die gleichen Kandelaber wie in der Vorstadt hier ein andersartiges, freudigeres und helleres Licht werfen. Das Mädchen ist auch von der Innenausstattung des Operngebäudes tief beeindruckt:

Als den Saal ein weißes Licht überströmte, begannen ringsherum tausend glitzernde Toiletten zu funkeln, schimmerten die weißen Schultern koketter Damen, und direkt vor uns flirrte, wie in Gold hochtoupiert, das Haar einer schönen Dame. Dies waren ganz neue Gesichter, aus Gott weiß welcher Welt. Auch nicht auf einem war Sorge oder Trauer; wohl gab es viele blasse und zerfurchte Gesichter; doch diese Gesichter lächelten und lachten, und Mimi sah, daß sie spitz und bleich wurden vor lauter Trunkenheit und lauter Glück. (Cankar 1995a: 12) 
Ähnlich wie Mme. Bovary, die sich in der Oper mit Donizettis Lucia di Lammermoor identifiziert, beginnt sich auch Mimi mit der Margarete aus Gounods Oper Faust zu vergleichen: „Auf der Bühne ist nicht mehr Margarete im weißen Gewand, mit jungfräulich geflochtenem, blondem Haar, Treue in den Augen und ein unschuldiges Lächeln auf den Lippen. Auf der Bühne ist Mimi ... Mimi lustwandelt im Garten, und Faust, der schöne junge Mann, hat sacht seinen Arm um ihre Taille geschlungen" (Cankar 1995a: 14). Mimi ist überzeugt, dass es sich um kein Märchen handelt, doch überlebt sie danach die Wiederkehr in die graue Realität nicht und stürzt sich aus dem dritten Stock in den Hof. Dem proletarischen Mädchen wird keine Apotheose zuteil wie im Fall Margaretes. Es ist bekannt, dass Cankar die Figur der Mimi nach dem Vorbild der dreizehnjährigen Steffi Löffler schuf, die er vielleicht auch in die Oper mitnahm. Gounods Faust stand zwischen den Jahren 1876 und 1909 auf Italienisch jedes Jahr auf dem Programm der Wiener Hofoper. ${ }^{4}$ Als Cankar sie im Jahr 1899 kennen lernte, war Steffi nur zwölf Jahre alt. Damals mietete er ein Kabinett bei Albina Löffler in der Lindauergasse 26. Fünfzehn Jahre später beschrieb er seinen ersten Kontakt zu seiner Vermieterin im Werk Ottakring, das jedoch Fragment blieb. Janko Kos (1972: 292) betonte, dass sich der schöpferischen Phantasie Cankars eine ganze Reihe von Motiven auftat, als er nach Ottakring zog. In seinem Aufsatz Kako sem postal socialist (Wie ich zum Sozialisten wurde) beschrieb er den Bezirk folgendermaßen:

Dort sah ich tagtäglich Dinge, die auch einen geistig flachen und im Herzen armen Menschen zum Nachdenken zwingen müßten. Das Unrecht der gesellschaftlichen „Ordnung“ stellte sich auf offener Straße zur Schau und gedieh in all seiner schamlosen Nacktheit. Im Winter, in Frost und Schnee, warf man die Familien stellenloser Arbeiter und ,selbstständiger", hungernder kleiner Gewerbetreibender auf die Gasse. Ich sah einen Mann, der bewußtlos auf dem Pflaster liegengeblieben war; man dachte, er sei vom Schnaps betrunken; als man den Rettungswagen gerufen hatte, stellte der Arzt fest, daß der zerlumpte Schwindsüchtige nicht wegen Trunkenheit zusammengebrochen war, sondern wegen Hunger. (Cankar 1994a: 30)

In Ottakring lebten im Jahr 1910 schon fast 11.000 Tschechen. Auch Albina Löffler, geb. Salbaba, war tschechisch-mährischer Abstammung, und Cankar beschrieb mehrmals - wie schon Govekar vor ihm - das Schicksal Wiener Proletarier tschechischer Herkunft. Jan Vymlatil in der Erzählung Brez doma (Ohne Heim) vermisst seine Heimat so sehr, dass er Selbstmord begeht (Cankar 1995b). Pavliček wird verhaftet, weil er am Geburtstag seines Sohnes eine Krone stahl. Es ist interessant, dass der Erzähler hier betont, dass Pavliček ihm gegenüber wohne und er manchmal in sein leeres Zimmer hineinschauen könne - als ob er hervorheben möchte, dass in Ottakring tragische Geschichten auf der Straße direkt vor dem Fenster geschehen.

${ }^{4}$ Die Wiener Hoftheater (Staatstheater). Ein Verzeichnis der aufgeführten und eingereichten Stücke mit Bestandsnachweisen und Aufführungsdaten 2. Wien: Hollinek, 1975, 285. 
Cankar beschrieb Ottakring oft, noch berühmter wurden jedoch seine Beschreibungen eines wichtigen Gebäudes im 18. Bezirk - in Währing. Er verewigte das dortige Haus der Barmherzigkeit, gestiftet von der Bruderschaft der heiligen Dreifaltigkeit, das man nur noch in der Literatur findet, da dessen ursprüngliches Gebäude während des Zweiten Weltkrieges zerstört wurde. Hier besuchte er in den Jahren 1901 und 1902 Steffis Schwester Amalia, ein todkrankes Mädchen, das ihm sehr nahe stand. Anhand dieser Eindrücke entstand sein wichtigstes Werk Hiša Marije Pomočnice (Das Haus der Barmherzigkeit, 1904). Aus der Perspektive eines Kindes sieht das Haus folgendermaßen aus:

Die Gänge waren leer, düster, die Schritte hallten von weitem um sie, als kämen und gingen Unsichtbare. Die hohen Fenster waren dunkel bemalt, doch blieb man stehen, hörte man Stimmen dahinter, seufzende, stöhnende, wie beim Rosenkranz in einer finsteren Kirche, spät in der Nacht, vor einem halberleuchteten Altar. Die Wände waren voll heiliger Bilder; rote Lichter brannten davor; welke Blumen hingen vom Rahmen. Malči sah diese Bilder an, ihre Augen waren wie hingezaubert, ihr graute und sie preßte sich an die Mutter. Auf dem ersten Bild war der Heilige Stefan gemalt; er war bis zum Gürtel nackt und kniete, den Kopf hielt er zurückgebeugt, die Augen waren zum Himmel gerichtet, die Hände vor der Brust gefaltet; der Körper war ganz weiß, sodaß er aus dem dunklen Bildnis hervortrat, als wäre er gemeißelt; lange Blutbäche rannen vom Gesicht, den Schultern, über den ganzen Körper, über die zarten weißen Hände; und das Gesicht war so ruhig, nur die Lippen schienen bewegt zu sein. Eine Unzahl roter, gewaltiger Arme erhob sich hinten, zur Rechten, zur Linken, wilde Gesichter spähten aus dem finsteren Hintergrund; der Heilige kniete ruhig auf einem Haufen blutigen Gesteins, seine Augen waren, als hätten sie jene Schönheit erblickt, nach der sie sich voller Tränen sehnten. Die blutigen Steine verwandelten sich in duftende rote Rosen. Auf dem zweiten Bild war eine Jungfrau, gekleidet in einen langen weißen Kittel. Auch ihr Gesicht war wunderbar ruhig. In den Händen hielt sie einen großen silbernen Teller, und darauf lagen ihre abgeschnittenen Brüste, weiße Jungfrauenbrüste. Weiße Lilien waren in ihrem Haar, und noch weißer war ihr Gesicht. Die Augen waren zum Himmel gerichtet, zum Altar gerichtet, auf dem sie ihre weißen Jungfrauenbrüste hingab ... Hinter ihr, im finsteren Hintergrund, drohten zwei wilde Gesichter. (Cankar 1996: 7-9)

Die beiden Heiligen, der Hl. Stefan und die Hl. Agathe, wurden nicht zufällig gewählt, da sie Märtyrer waren. Der gesteinigte Stefan und die verstümmelte Agathe, die wunderbar ruhige Gesichter haben, werden im Hintergrund von dunklen Gestalten umgeben. Später wird auch die Hl. Agnes erwähnt, die als Heilige der Kinder und Hüterin der Unbeflecktheit gilt. Die Jugend der Mädchen wurde im heimischen Umfeld geschändet (auch der alte Löffler war ein krimineller Mensch, der seine Familie belästigte), was bedeutet, dass sie eine Art Märtyrerinnen waren. Im kirchlichen Umfeld, im Umgang mit der sakralen Ikonographie, dem desakralisierten Zuhause entrissen, werden die Mädchen erneut konsakralisiert und werden wieder unbefleckt. Der Wunsch nach Konsakralisierung verband sich mit der Flucht aus der Realität. Das Krankenhaus Haus der Barmherzigkeit war also eine Art Zufluchtsort vor der Grausamkeit der Welt.

Bei Cankar findet man nur selten genaue Beschreibungen der Sehenswürdigkeiten Wiens, eine wichtige Ausnahme ist die Skizze Dunaj poleti (Wien im Sommer). Auf vier Seiten schildert der Autor einfallsreich einige der wichtigsten Wiener Institutionen und ihre Vertreter im Jahr 1900. Auch das ist Wien, wie Cankar es erlebt 
hatte. Während im „Venedig“ im Prater ${ }^{5}$ Erfolgsstücke aufgeführt werden, hat Paul Schlenther, Direktor des Burgtheaters, große Schwierigkeiten, da ihm sogar seine Frau vorwirft, unbrauchbar zu sein, weil sein Theater leer ist: „[D]u kamst nach Wien, und in ein paar Jahren ruiniertest du glücklich das bedeutendste deutsche Theater" (Cankar 1994b: 53). So wie viele Kritiker tat auch Cankar Schlenther Unrecht, da der Direktor vor allem Novitäten aufführte. Es gab tatsächlich weniger Vorstellungen, doch gerade im Jahr 1900 war Schlenther selbst Regisseur der Gioconda von D’Annunzio, wo sogar Eleonora Duse auftrat. ${ }^{6}$ Cankar erwähnt in der Skizze auch den damaligen Wiener Bürgermeister Karl Lueger und seinen Stellvertreter Josef Strobach, der im Bezirk Margarethen angeblich hochnäsig die Proletarier beobachtet und sie als „G'sind'l“ bezeichnet habe. Der Erzähler schleicht sich danach in die Sitzung des Ministerrates ein, wo sich auf die Glatze des Ministerpräsidenten Ernst Koerber eine Fliege setzt. Die zentrale Botschaft der Erzählung ist, dass Minka, „die Frau aller Frauen“, nach Wien kam: „Meine Braut und ich haben für das slowenische Volk auf jeden Fall mehr getan, als Minister Koerber und der fromme Call“. (Cankar 1994b: 56)

\section{Slavko Grum / Slavko Grom}

Im Jahr 1919, nach dem Zerfall der Monarchie, begab sich noch ein slowenischer Literat zum Studium der Medizin nach Wien - Slavko Grum, der zuerst auch ein Philosophiestudium in Erwägung zog. Zuerst wohnte er in Währing, später in Hernals, sogar in der gleichen Straße (Hernalser Hauptstraße), in der drei Jahrzehnte davor der gescheiterte Mediziner Govekar wohnte. In den ersten Briefen aus Wien klagte er über die hohen Preise, Hunger und jeden Tag härtere Kälte, da nirgendwo geheizt wurde (Grum 1976a: 11). In späteren Jahren beschrieb er den Stadtpark in einem Brief an Joža Debelak, die er sich neben sich in Wien vorstellte, folgendermaßen:

Als du kamst, war es auf einmal ein Festtag und ich ging mit dir in den Park. Der Park ist nicht schön, verludert, mit dem tödlichen Modergestank, die Beete noch voller Blumen. Diese Menschen schänden alles. Warum pflanzen sie jetzt noch Blumen? Warum lassen sie sie nicht sterben? Wie geschminkte Hühner sehen diese Astern aus. Ich setzte mich auf eine abgelegene Bank, rundherum nur totes Laub. Damit ich mit dir allein wäre. Irgendwo in der Ferne röchelten die Straßen und bellten die Wagen - ich aber war so selig alleine mit deinen samtenen Händen (Grum 1976a: 19).

5 Gabor Steiner hat im Jahr 1895 im Wiener Prater den Themenpark „Venedig“ mit echten Kanälen, Gondolieri und Palästen errichtet (Autengruber 2008: 47).

6 Schlenthers Name taucht sechs Jahre später auch in der berühmten Theaterdiskussion auf. Cankar meinte, dass man Govekar aus dem Theater verbannen sollte, da er Sudermann favorisierte. Govekar erwiderte, dass man dann auch Schlenther verbannen sollte, da er den gleichen Dramatiker aufführe (Moravec 1972: 341). 
Mit Grums Hervorhebungen des Hässlichen und des Vermoderten trat in die Geschichte der slowenischen Beschreibungen Wiens auch der Expressionismus ein. Etwas später gestand Grum Debelak, dass ihm das Klima einer Kaschemme so „unendlich gut tue", wo außer ihm nur noch Fiaker und Prostituierte mit ihren Zuhältern verkehrten (Grum 1976a: 23). Lado Kralj (1976: 402) ist der Meinung, dass in dieser Kaschemme auch Grums berühmteste Wiener Kurzgeschichte Pivnica Pri deseti Mariji (Das Weinhaus zur 10er Marie) spiele. Der Schriftsteller beschrieb die dortige Stimmung folgendermaßen:

Ich sitze am Fenster und warte, bis die Luft dort draußen sich in Schneeflocken bewegt. Der Zigarettenrauch lockert meine Gedanken in violette Bänder, Gelächter, Klang der Krüge plätschert gegen mich. Alle Gesichter, die um mich herum in der verrauchten Ferne schwimmen, sind aufgedunsen und angeschwollen vor Glück. Doch steckt in ihnen etwas Schreckliches. Sie reden zu laut, zu laut schieben sie die Stühle herum. Es scheint, als ob sie vor etwas Angst hätten (Grum 1976b: 88).

Obwohl Grum wahrscheinlich wirklich die Stimmung in Ottakring beschreibt, hat seine Kurzgeschichte nichts mit dem realen Weinhaus zur 10er Marie zu tun, welches seinen Namen nach der Hausnummer und der schönen Wirtstochter Marie bekam und wo vor allem berühmte Künstler und sogar der Thronfolger verkehrten. In diesem Ottakringer Umfeld schildert der Autor die unglücklichen Schicksale eines Jungen, der Blumen verkauft, und eines Mädchens, das ihrer Mutter - einer Prostituierten - weggenommen wurde.

Fast achtzig Jahre nach Grums Abschied von Wien haben seine Lebensgeschichte und sein Opus den bekannten slowenischen Dichter Milan Dekleva dazu angeregt, den pseudobiographischen Roman Zmagoslavje podgan (Der Rattensieg) über Slavko Grom zu schreiben, worin Grom mit Freud und Wagner-Jauregg plaudert, aber auch mit Menschen, die der reale Grum kannte. Dekleva unterstreicht, dass die Personen, auch die, die historische Namen tragen, reine poetische Erfindung seien. Es haben viele der originellen Grumschen Motive Eingang in diesen Roman gefunden, die freilich umgearbeitet wurden. Es kommt das Weinhaus zur 10er Marie vor, das von Dekleva als „Zufluchtsort der vorstädtischen Trinker und Obdachlosen, kleiner Diebe, ältlicher Prostituierter und des Lumpenproletariats" beschrieben wird (Dekleva 2006: 28). Dekleva schildert auch einen Spaziergang am Ring:

Als [Grom] den Ring überquert, wirbelt zwischen den Kutschen, Kinderwagen und Automobilen der Wind, macht sie auf den sich nähernden Herbst aufmerksam, streift sie, verlässt sie und verfängt sich in den Ästen der Bäume im Park vor dem Rathaus. Das Laub tänzelt in die Luft; der Tod tanzt. Leichtes Laub; leichter Tod. Spätnachmittag mit langen Schatten, langen Blicken in die Leere des Bleihimmels (Dekleva 2006: 9).

So kommt Wien auch in einem der besten slowenischen Romane der letzten Zeit vor. 


\section{Schluss}

Aus der vorliegenden Untersuchung, die sicherlich nicht alle Textbeispiele abdeckt, ${ }^{7}$ ist ersichtlich, dass slowenische Schriftsteller den Wiener Parks viel Aufmerksamkeit widmeten. Bereits bei Valjavec und Levstik werden die Parks als schöne Gärten dargestellt, wo es von schönen Mädchen wimmelt. Im Unterschied zu Govekar, dem bei der impressionistischen Beschreibung des Volksgartens keine Kleinigkeit entgeht, ist der eher allgemein beschriebene Park bei Cankar ein Symbol des Unerreichbaren. Das Parkbild veränderte sich vollkommen mit dem Expressionismus, da Grum nur das Vermoderte wahrnahm. Govekar jedoch führte in die slowenische Literatur auch das Leiden der tschechischen Proletarier in Hernals ein, ihr großer Ottakringer Dichter war jedoch Ivan Cankar. Seiner Ottakringer Motivik folgte auch Grum. Im Gegensatz zu den anderen Autoren suchte Cankar in Wien auch den symbolischen und kurzfristigen Zufluchtsort für erschöpfte Proletarier, den er in der Oper und im Haus der Barmherzigkeit fand.

Übersetzt von Amalija Maček

\section{Literatur}

Autengruber 2008:

Balk 1997:

Bernik 1998:

Cankar 1968a:

Cankar 1968b:

Cankar 1969:

Cankar 1970:

Cankar 1994a:

Cankar 1994b:

Cankar 1994c:

Cankar 1995a:

Cankar 1995b:
Peter Autengruber, Parks und Gärten in Wien, Wien

Claudia Balk, Pathos und Komik. Von „Der Sandrock“ zur Adele, München

France Bernik, Ivan Cankar und Wien. Österreich-slowenische Literaturbeziehungen, Wien, 293-304

Ivan Cankar, Ruhebedürfnis, Zbrano delo 2, Ljubljana, 139

Ivan Cankar, Iz predmestja, Zbrano delo 8, Ljubljana, 135-149

Ivan Cankar, Tisti lepi večeri, Zbrano delo 7, Ljubljana, 7-13

Ivan Cankar, Pisma I, Zbrano delo 26, Ljubljana

Ivan Cankar, Wie ich zum Sozialisten wurde, Vor dem Ziel. Aus dem Slowenischen übersetzt von Erwin Köstler, Klagenfurt/Celovec, 2135

Ivan Cankar, Wien im Sommer, Vor dem Ziel. Aus dem Slowenischen übersetzt von Erwin Köstler, Klagenfurt/Celovec, 50-57

Ivan Cankar, Vor dem Ziel. Vor dem Ziel, Aus dem Slowenischen übersetzt von Erwin Köstler, Klagenfurt/Celovec, 58-78

Ivan Cankar, Mimi, Pavličeks Krone. Literarische Skizzen aus Wien. Aus dem Slowenischen übersetzt von Erwin Köstler, Klagenfurt/Celovec, 7-17

Ivan Cankar, Ohne Heim, Pavličeks Krone. Literarische Skizzen aus Wien. Aus dem Slowenischen übersetzt von Erwin Köstler, Klagenfurt/Celovec, 48-53

\footnotetext{
${ }^{7}$ Gerade in letzter Zeit hat Lado Kralj in seinem Buch Kosec koso brusi eine Novelle mit dem Titel Narrenturm veröffentlicht, die den Wiener Stadtplan tatsächlich in Worte verwandelt, da die Geschichte in unterschiedlichen Stadtteilen spielt, vom Museumsquartier bis zum AKH. Die beiden slowenischen Protagonisten fragen auch oft auf U-Bahn Stationen nach Auskunft (Kralj 2010).
} 
Cankar 1996:

Cigler 1936:

Claricini 1996:

Dekleva 2006:

Govekar 1896a:

Govekar 1896b:

Govekar 1896c:

Govekar 1897:

Govekar 1978:

Grum 1976a:

Grum 1976b:

Jurčič 1982:

Kos 1972:

Kralj 1976:

Kralj 2010:

Levec 1967:

Levstik 1948:

Levstik 1952:

Moravec 1972:

Simonek 2004:

Stritar 1955:

Stritar 1957:

Trdina 1948:

Valjavec 1900:
Ivan Cankar, Das Haus der Barmherzigkeit. Aus dem Slowenischen übersetzt von Erwin Köstler, Klagenfurt/Celovec

Janez Cigler, Sreča v nesreči, Celje

Maria Vera Claricini, Cankars Wien - ein Ausschnitt der Stadt. Das Bild Wiens in der slowenischen Literatur, Wien als Magnet? Schriftsteller aus Ost-, Ostmittel- und Südosteuropa über die Stadt, hrsg. v. Gertraud Marinelli-König und Nina Parlora, Wien, 393-435

Milan Dekleva, Zmagoslavje podgan, Ljubljana

Fran Govekar, V krvi, Ljubljanski zvon XVI

Fran Govekar, ,Socijalist!“, Ljubljanski zvon XVI, 156-165; 229-241

Fran Govekar, Vzor, Ljubljanski zvon XVI, 682-693; 742-746

Fran Govekar, O te ženske, Gorica

Fran Govekar, Pisma I, Ljubljana

Slavko Grum, Zbrano delo 2, Ljubljana

Slavko Grum, Pivnica "Pri deseti Mariji", Zbrano delo 1, Ljubljana, 88-90

Josip Jurčič, Pisma z Dunaja, Zbrano delo 10, Ljubljana, 208-218

Janko Kos, Opombe. Ivan Cankar. Zbrano delo 11, Ljubljana, 285340

Lado Kralj, Opombe. Slavko Grum. Zbrano delo 1, Ljubljana, 367496

Lado Kralj, Kosec koso brusi, Ljubljana

Fran Levec, Pisma I, Ljubljana

Fran Levstik, Dunajčanki, Zbrano delo 1, Ljubljana, 212-214

Fran Levstik, Meniška cerkev in dunajski vrt, Zbrano delo 2, 64-66

Dušan Moravec, Opombe. Ivan Cankar. Zbrano delo 15, Ljubljana, $291-394$

Stefan Simonek, Svet dunajskih parkov pri Ivanu Cankarju in Ivu Vojnoviću, Jezik in slovstvo XLIX, 81-90

Josip Stritar, Popotna pisma, Zbrano delo 6, Ljubljana

Josip Stritar, Zbrano delo 9, Ljubljana

Janez Trdina, Spomini. 2. del, Zbrano delo 2, Ljubljana

Matija Valjavec, Poezije, Ljubljana

A bstra ct: Vienna and Slovene Literature. Our research shows that Slovene writers devoted considerable attention to Viennese parks. Valjavec and Levstik described them as beautiful gardens teeming with beautiful young women. Unlike Govekar, who did not miss even the smallest detail in his impressionist description of Volksgarten, Cankar more generally described parks as a symbol of something unattainable. The attitude towards parks changed completely with Expressionism, with Grum focusing only on decay. Govekar introduced into Slovene literature descriptions of the suffering of the Czech working class in Hernals, but their great Ottakring poet was Cankar. Grum probably followed his Ottakring social motifs. Unlike others, Cankar also sought in Vienna a short-term symbolic refuge for the battered working class, which he found in the Viennese opera and in the Ward of our Lady of Mercy.

K e y w o r d s : Vienna, Slovene literature, Fran Govekar, Ivan Cankar

\author{
Tone Smolej \\ Oddelek za primerjalno književnost in literarno teorijo \\ Filozofska fakulteta Univerze v Ljubljani \\ Aškerčeva 2 \\ 1000 Ljubljana, Slowenien \\ tone.smolej@guest.arnes.si
}

